\title{
Association of matrix metalloproteinase-2 gene promoter polymorphism with myocardial infarction susceptibility in a Mexican population
}

\author{
IVAN DELGADO-ENCISO ${ }^{1 *}$, NELIDA A. GONZALEZ-HERNANDEZ ${ }^{1}$, LUZ M. BALTAZAR-RODRIGUEZ ${ }^{1}$, \\ REBECA O. MILLAN-GUERRERO' ${ }^{2}$, OSCAR NEWTON-SANCHEZ ${ }^{1}$, ALFONSO BAYARDO-NORIEGA ${ }^{2}$, \\ ALFONSO ALEMAN-MIRELES ${ }^{2}$, IRMA G. ENRIQUEZ-MALDONADO ${ }^{3}$, MA J. ANAYA-CARRILLO ${ }^{1}$, \\ AUGUSTO ROJAS-MARTINEZ ${ }^{4}$ and ROCIO ORTIZ-LOPEZ ${ }^{4}$ \\ ${ }^{1}$ School of Medicine, Universidad de Colima, Av. Universidad 333, Colonia Las Viboras, CP 28040, Colima, Col., Mexico \\ ${ }^{2}$ General Hospital $N^{\circ}$ 1, Instituto Mexicano del Seguro Social, Colima, Zaragoza 377, Colonia, CP 28040, Mexico \\ ${ }^{3}$ Hospital Regional Universitario, Secretaria de Salud del Estado de Colima, Km 2.0 Carretcra \\ Colima-Guadalajara, CP 28019, Colima, Mexico \\ ${ }^{4}$ School of Medicine, Universidad Autonoma de Nuevo Leon, Av. Madero Y Aguirre Peqeño, Mitras Centro, \\ CP 64460, Monterrey Nuevo Leon, Mexico
}

\begin{abstract}
Introduction
Previous studies have revealed elevated levels of matrix metalloproteinase-2 (MMP-2) in atherosclerotic plaques of coronary arteries and in plasma after the onset of acute myocardial infarction (AMI) (Kai et al. 1998). An MMP-2 single nucleotide polymorphism, $-1306 \mathrm{~T}>\mathrm{C}$, displayed strikingly high promoter activity of the $\mathrm{C}$ allele (Price $e t a l$. 2001). The present study analysed whether or not the MMP$2-1306 \mathrm{~T}>\mathrm{C}$ polymorphism contributed to the development of AMI in a Mexican population. An increased probability of AMI associated with the MMP-2 CC genotype was found. AMI risk associated with the CC genotype was more pronounced in obese or hypertensive individuals. The data suggest that MMP-2 -1306T $>$ C polymorphism contributes to AMI development in the population studied.

Myocardial infarction is the major cause of death by disease in the world (Ciruzzi et al. 2003). The most important risk factors for AMI are hypercholesterolemia, high blood pressure, diabetes, obesity, smoking and family history of coronary heart disease. Atherosclerosis of the coronary arteries is the predominant AMI mechanism. Atherosclerotic plaque growth occurs through structural changes which allow the accumulation of cells, extracellular matrix and lipids in the intimate layer of the diseased artery. The rupture or erosion of the fibrous layer of the atheromatous plaque gives rise to thrombosis, which is the factor setting off, a chain of events leading to AMI (Newby 2005; Tedgui and Mallat 2006).
\end{abstract}

*For correspondence. E-mail: ivan_delgado_enciso@ucol.mx.
AMI physiopathology is complex at the molecular level and involves a wide variety of proteins, including the matrix metalloproteinases (MMPs). Experiments suggest that MMP action can facilitate the formation of atheromatous plaque (Tedgui and Mallat 2006). Additionally, some MMPs (MMP-2, MMP-3 and MMP-9) specifically attack type IV collagen, laminin and fibronectin, which are the major components of the basal lamina around blood vessels (Newby 2005). There is evidence that the action of MMP enzymes weakens the arterial wall, contributing to the destabilizing and rupture of atheromatous plaque, leading to AMI (Newby 2005; Tedgui and Mallat 2006).

Elevated MMP-2 levels have been detected in the plasma of patients with angina pectoris (Kai et al. 1998). Overexpressed MMP-2 has also been found directly in atherosclerotic plaques of coronary arteries (Pasterkamp et al. 2000). MMP-2 blood levels were shown to increase gradually after the onset of AMI, with maximum elevation on day 21 after onset (Hojo et al. 2001). High levels of MMP-1, MMP -9, and MMP-13 have also been detected in post-infarction phases (Hlatky et al. 2007; Suzuki et al. 2008). These observations show the association of MMPs with physiopathological processes of AMI.

MMP expression can vary among individuals due to genetic differences, which could influence susceptibility to vascular disease. At present, there are only a few polymorphisms within the MMP genes that are clearly associated with AMI (MMP-3 -1171 5A/6A, MMP-9 -1562C/T and 279R/Q) (Abilleira et al. 2006). However, studies on MMP

Keywords. acute myocardial infarction; cardiovascular risk factors; human genetics; matrix metalloproteinases. 
genetic variations in AMI are scarce. Price et al. (2001) identified the $-1306 \mathrm{~T}>\mathrm{C}$ polymorphism of the MMP-2 promoter. This base transition is located in the CCACC box of the Sp1binding site. The -1306CC genotype causes elevated enzymatic MMP-2 expression and activity, and could be an AMI risk factor. The present case-control study analyses the association between the MMP-2 -1306C $>$ T polymorphism and the development of AMI in a population in western Mexico.

\section{Materials and methods}

\section{Study population}

Written informed consent was obtained from all the subjects who participated in this study, and the study protocol was approved by the institutional review board of the School of Medicine of the University of Colima and the IMSS Hospital General (HG) $\mathrm{N}^{\circ} 1$. The study conformed to the principles of the World Medical Association's Declaration of Helsinki.

Between January 2006 and May 2007, a total of 98 AMI patients (case group) were recruited from a public health system hospital (HG $\mathrm{N}^{\circ} 1$ of the IMSS) in the city of Colima, Mexico. The control group comprised of 163 nonhospitalized individuals with no medical history of ischemic vascular disease (corroborated clinically and by electrocardiography), who were seen in family medicine consultations in the same public hospital at the same point of time. The criteria for selecting subjects for the control group were same sex and similar age (not more than five-years difference) as patients in the case group (1-2 control subjects per one case subject). The control group subjects had come to the hospital for routine check-ups, preventive or early diagnosis and treatment measures for diabetes, high blood pressure or some acute ailment not requiring hospitalization. Individuals suffering from autoimmune, thyroid, neoplastic, or hepatic (such as cirrhosis) diseases or presenting with chronic infections were excluded from this study. All study participants were non-blood-related Mexican mestizo subjects from the state of Colima, Mexico. A case history was elaborated for each participant through interviews and data obtained from official medical records.

\section{Definitions}

AMI diagnosis was made by a cardiologist based on World Health Organization (WHO) criteria. Arterial hypertension was considered to be present if the patient had a history of high blood pressure or presented with blood pressure values above 140/90 mm $\mathrm{Hg}$ one week after the event. High blood pressure was also considered present in patients receiving antihypertensive medication (Hsieh et al. 2005). Diabetes mellitus was considered to be present when fasting blood glucose concentrations were over $1260 \mathrm{mg} / \mathrm{L}$ or if a history of diabetes was reported (including patients receiving diabetes medication). Hyperlipidemia was defined as fasting total serum cholesterol over $2000 \mathrm{mg} / \mathrm{L}$ or triglycerides over $2000 \mathrm{mg} / \mathrm{L}$, and was considered to be present in all patients receiving lipid-lowering medications. Smoking was defined as the smoking of 100 or more cigarettes during the patient's lifetime (Medina-Mora et al. 2002). An individual was considered to be overweight or obese if body mass index was over 25 and 30, respectively.

\section{Polymorphism analysis}

Peripheral venous blood $(2 \mathrm{~mL})$ was drawn from participants and stored in EDTA tubes for no longer than a week at $4^{\circ} \mathrm{C}$ until DNA extraction was carried out (phenolchloroform extraction and DNA precipitation with absolute ethanol). MMP-2 polymorphism was studied by the PCR-RFLP method, as previously described (GonzalezHernandez et al. 2008).

\section{Statistical analysis}

Evaluation of Hardy-Weinberg equilibrium was performed by comparing observed and expected genotypes, using chisquare analysis. Chi-square test (the Fisher exact test) was used to examine differences between cases and controls when two nominal variables were compared, and the Student $t$-test was used to compare the mean values of the measurement variables (age or BMI, both normally distributed) The association between the MMP-2 polymorphism and risk of AMI was estimated by odds-ratios (OR) and $95 \%$ confidence intervals (c.i.) (Crosstabs procedure), which were calculated controlling others risk factors using a Mantel-Haenszel analysis. All statistical analyses were performed with SPSS version 11.0 software (SPSS, Chicago, USA).

\section{Results}

The principal clinical characteristics, such as genotype frequency, are shown in table 1 . Of all the clinical characteristics, hyperlipidemia was the only one in which there was a significant difference between cases and controls. MMP-2 $-1306 \mathrm{C}$ allele frequency was 0.78 and 0.82 , for the control and case groups, respectively $(P=0.18)$. The distribution of MMP-2 genotypes in the control group (table 1) was in Hardy-Weinberg equilibrium $(P=0.65)$. Since TT genotype was rare $(4.3 \%$ of the case group and $8.2 \%$ of the control group), it was combined with the CT genotype as the variant group for subsequent analysis.

Because the proportion of hyperlipidemia was different between case and control groups, all OR analyses were carried out controlling for that variable. The adjusted OR for subjects carrying the CC genotype was 2.05 (95\% c.i.: $1.09-3.85, P=0.02$ ) for AMI. Effects of the CC genotype were additionally examined in subgroups of individuals with high blood pressure, diabetes and obesity (table 2). The distribution of MMP-2 genotypes in the control subgroups (high blood pressure, diabetic or obese patients) were in HardyWeinberg equilibrium. It was found that increased probability of AMI associated with the MMP-2 CC genotype was more pronounced in obese or hypertensive subjects. 
Table 1. Distribution of selected characteristics by case-control status.

\begin{tabular}{lccc}
\hline Variables & $\begin{array}{c}\text { Control } \\
n=163(\%)\end{array}$ & $\begin{array}{c}\text { Case } \\
n=98(\%)\end{array}$ & $P$ \\
\hline Age (years) & $61.4 \pm 9.2$ & $61.7 \pm 9.9$ & 0.63 \\
Sex & & & \\
$\quad$ Female & $66(40.5)$ & $35(35.7)$ & 0.51 \\
$\quad$ Male & $97(59.5)$ & $63(64.3)$ & \\
Smoking & $82(50.3)$ & $57(58.2)$ & 0.25 \\
Diabetes 2 & $69(42.3)$ & $43(43.9)$ & 0.89 \\
Hypertension & $102(62.6)$ & $61(62.2)$ & 1.00 \\
Dislypidemia & $25(15.3)$ & $62(63.3)$ & $0.00 *$ \\
BMI, kg/m ${ }^{2}$ & $28.2 \pm 4.4$ & $28.9 \pm 4.7$ & 0.55 \\
Obesity & $46(28.2)$ & $34(34.7)$ & 0.33 \\
Genotype & & & \\
TT & $7(4.3)$ & $8(8.2)$ & 0.27 \\
CT & $60(36.8)$ & $19(19.4)$ & $0.00 *$ \\
CC & $96(58.9)$ & $71(72.4)$ & $0.04 *$ \\
\hline
\end{tabular}

*Significant difference.

Table 2. Odds ratio (OR) for effects of MMP-2 -1306 polymorphism on acute myocardial infarction in hypertensive, diabetic and obese patients.

\begin{tabular}{lcccc}
\hline Sample & $\begin{array}{c}\text { Genotype }(n) \\
\text { CT/TT/CC }\end{array}$ & $\begin{array}{c}\text { Adjusted OR** } \\
(\text { c.i. 95\%) }\end{array}$ & $P$ \\
\hline Control & $66^{*}$ & 97 & $2.05(1.09-3.85)$ & 0.025 \\
$\quad$ Case & 27 & 71 & & \\
Hypertensive subjects & & & & \\
$\quad$ Control & $43^{*}$ & 59 & $3.03(1.25-7.32)$ & 0.014 \\
$\quad$ Case & 16 & 45 & & \\
Diabetic subjects & & & & \\
$\quad$ Control & $26^{*}$ & 43 & $2.28(0.84-6.18)$ & 0.103 \\
$\quad$ Case & 12 & 31 & & \\
Obesity subjects & & & & \\
$\quad$ Control & $21^{*}$ & 25 & $3.59(1.20-10.74)$ & 0.022 \\
$\quad$ Case & 6 & 28 & & \\
\hline
\end{tabular}

*Reference; **adjusted for hyperlipidemia.

\section{Discussion}

Results from the present study showed that the MMP-2 1306 CC genotype increased AMI risk (OR 2) in the population studied. Previously, Vasku et al. (2004) had found that a haplotype made up of four MMP-2 promoter polymorphisms (-1575G, -1306C, -790T and -735C) was associated with coronary triple-vessel disease. Although Vasku et al. (2004) found no direct association between the MMP-2 -1306 polymorphism and cardiac ischemia, a haplotype containing the $-1306 \mathrm{C}$ allele was found to be associated with the disease. This is congruent with our results. However, it is important to mention that our study and that of Vasku et al. (2004) were carried out on populations of different ethnic origins and that not all the patients analysed by Vasku $e t$ al. (2004) presented with AMI. This could explain why the MMP-2 -1306 polymorphism, itself, was not associated with ischemic cardiopathy (coronary triple-vessel disease) in the study of Vasku et al. (2004).

In a recent study by Horne et al. (2007), on different MMP polymorphisms in AMI, various MMP-1/MMP-3 and MMP-9 genotypes were associated with the disease. However, MMP-2 -1306 polymorphism genotypes were not associated with the development of AMI, unlike in our study. This could be due to the different ethnic origins of the respective study populations. Ninety per cent of the individuals analysed by Horne et al. (2007) were from a Caucasian population of the United States, while our study included only a western Mexican mestizo population. Although there can be discrepancies at a genetic level as to the importance of the role of MMP-2 polymorphisms as AMI risk factors, the fact that MMP-2 intervene in the processes that trigger and /or signal AMI evolution has clearly been demonstrated (Pasterkamp et al. 2000; Hojo et al. 2001; Newby 2005; Tedgui and Mallat 2006).

There is an elevation of MMP-2 levels in different stages of AMI physiopathology. This enzyme is elevated in atherosclerotic plaques of coronary arteries (Pasterkamp et al. 2000) as well as in plasma after the onset of AMI (Hojo et al. 2001). Additionally, mice with cardiac-specific transgenic expression of active MMP-2 have a larger infarction size compared to wild-type hearts (Zhou et al. 2007). MMP2 -1306CC genotype increases MMP-2 expression in vitro and this increase could be related to AMI through two processes: (i) by facilitating leukocyte and lipoprotein infiltration into the endothelium, which is necessary for the development of atherosclerosis, and (ii) by weakening the arterial wall, which can destabilize and break atheromatous plaque, leading to AMI (Newby 2005; Tedgui and Mallat 2006).

It was also observed that the presence of the CC genotype in hypertensive or obese patients increased AMI risk more than three-fold. In diabetic patients, the OR for the genotype remained stable and did not demonstrate statistically significant changes. Chronically hypertensive patients have presented with an increase of MMP-2 expression and activation (Yasmin et al. 2005), while obese patients have presented with an elevation of leptin (an endocrine hormone) which enhances MMP-2 expression in coronary vascular cells (Park et al. 2001). It is probable that MMP-2 elevation by the -1306CC genotype becomes more relevant and represents more AMI risk when another factor that increases the enzyme is present, as occurs in obese or hypertensive patients. Similar results were found for ischemic stroke: a clear association between -1306CC genotype and IS was detected, but only in hypertensive patients (Gonzalez-Hernandez et al. 2008).

The MMP-2 -1306CC genotype acts as an AMI risk factor in more than half of the patients studied. MMP inhibition has already been proposed for the treatment of cardiovascular disease (Peterson 2006). The results of the present study 
lend support to the idea that MMP-2 inhibition could become a therapeutic target for AMI prevention, at least in the population studied.

An important characteristic of the present study was that AMI diagnosis was carried out based on WHO criteria, which does not detect very small infarcts, unlike the criteria recommended by the European Society of Cardiology and the American College of Cardiology (Alpert et al. 2000). This must be taken into account when comparing the present study with similar studies.

The present study showed that the MMP-2 -1306CC genotype increased the risk of developing acute myocardial infarction. More such studies on different populations need to be carried out.

\section{References}

Abilleira S., Bevan S. and Markus H. S. 2006 The role of genetic variants of matrix metalloproteinases in coronary and carotid atherosclerosis. J. Med. Genet. 43, 897-901.

Alpert J. S., Thygesen K., Antman E. and Bassand J. P. 2000 Myocardial infarction redefined - a consensus document of The Joint European society of cardiology/American college of cardiology committee for the redefinition of myocardial infarction. $J$. Am. Coll. Cardiol. 36, 959-969.

Ciruzzi M., Schargrodsky H., Pramparo P., Rivas Estany E., Rodriguez Naude L., de la Noval Garcia R. et al. 2003 Attributable risks for acute myocardial infarction in four countries of Latin America. Medicina (Buenos Aires) 63, 697-703.

Gonzalez-Hernandez N. A., Millan-Guerrero R. O., Ceja-Espiritu G., Baltazar-Rodriguez L. M., Rodriguez-Salazar R., OlmedoBuenrostro B. A. et al. 2008 A polymorphism in the matrix metalloproteinase-2 (MMP-2 -1306T $>$ C) gene promoter is associated with high risk of ischemic stroke in hypertensive patients. Genes Genom. 30, 533-540.

Hlatky M. A., Ashley E., Quertermous T., Boothroyd D. B., Ridker P., Southwick A. et al. 2007 Atherosclerotic disease, vascular function and genetic epidemiology (ADVANCE) study. Matrix metalloproteinase circulating levels, genetic polymorphisms, and susceptibility to acute myocardial infarction among patients with coronary artery disease. Am. Heart J. 154, 1043-1051.

Hojo Y., Ikeda U., Ueno S., Arakawa H. and Shimada K. 2001 Expression of matrix metalloproteinases in patients with acute myocardial infarction. Jpn. Circ. J. 65, 71-75.

Horne B. D., Camp N. J., Carlquist J. F., Muhlestein J. B., Kolek M. J., Nicholas Z. P. and Anderson J. L. 2007 Multiplepolymorphism associations of 7 matrix metalloproteinase and tissue inhibitor metalloproteinase genes with myocardial infarction and angiographic coronary artery disease. Am. Heart J. 154, 751-758.
Hsieh K., Lalouschek W., Schillinger M., Endler G., Reisinger M., Janisiw M. et al. 2005 Impact of _ENaC Polymorphisms on the risk of ischemic cerebrovascular events: a multicenter casecontrol study. Clin. Chem. 51, 952-956.

Kai H., Ikeda H., Yasukawa H., Kai M., Seki Y., Kuwahara F. et al. 1998 Peripheral blood levels of matrix metalloproteases-2 and -9 are elevated in patients with acute coronary syndromes. $J$. Am. Coll. Cardiol. 32, 368-372.

Medina-Mora M. A., Peña-Corona M. P., Cravioto P., Villatoro J. and Kuri P. 2002 Del tabaco al uso de otras drogas: ?el uso temprano de tabaco aumenta la probabilidad de usar otras drogas? Salud Publica Mex. 44, S109-S115.

Newby A. C. 2005 Dual role of matrix metalloproteinases (matrixins) in intimal thickening and atherosclerotic plaque rupture. Physiol. Rev. 85, 1-31.

Park H. Y., Kwon H. M., Lim H. J., Hong B. K., Lee J. Y., Park B. E. et al. 2001 Potential role of leptin in angiogenesis: leptin induces endothelial cell proliferation and expression of matrix metalloproteinases in vivo and in vitro. Exp. Mol. Med. 33, 95102.

Pasterkamp G., Schoneveld A. H., Hijnen D. J., de Kleijn D. P., Teepen H., van der Wal A. C. and Borst C. 2000 Atherosclerotic arterial remodeling and the localization of macrophages and matrix metalloproteases 1,2 and 9 in the human coronary artery. Atherosclerosis 150, 245-253.

Peterson J. T. 2006 The importance of estimating the therapeutic index in the development of matrix metalloproteinase inhibitors. Cardiovasc. Res. 69, 677-687.

Price S. J., Greaves D. R. and Watkins H. 2001 Identification of novel, functional genetic variants in the human matrix metalloproteinase- 2 gene: role of $\mathrm{Sp} 1$ in allele-specific transcriptional regulation. J. Biol. Chem. 276, 7549-7558.

Suzuki H., Kusuyama T., Sato R., Yokota Y., Tsunoda F., Sato T. et al. 2008 Elevation of matrix metalloproteinases and interleukin6 in the culprit coronary artery of myocardial infarction. Eur. J. Clin. Invest. 38, 166-173.

Tedgui A. and Mallat Z. 2006 Cytokines in atherosclerosis: pathogenic and regulatory pathways. Physiol. Rev. 86, 515-581.

Vasku A., Goldbergová M., Izakovicová Holla L., Sisková L., Groch L., Beránek M. et al. 2004 A haplotype constituted of four MMP-2 promoter polymorphisms (-1575G/A, -1306C/T, $-790 \mathrm{~T} / \mathrm{G}$ and $-735 \mathrm{C} / \mathrm{T})$ is associated with coronary triple-vessel disease. Matrix Biol. 22, 585-591.

Yasmin, McEniery C. M., Wallace S., Dakham Z., Pulsalkar P., Maki-Petaja K. et al. 2005 Matrix metalloproteinase-9 (MMP-9), MMP-2, and serum elastase activity are associated with systolic hypertension and arterial stiffness. Arterioscler. Thromb. Vasc. Biol. 25, 372-378.

Zhou H. Z., Ma X., Gray M. O., Zhu B. Q., Nguyen A. P., Baker A. J., Simonis U. et al. 2007 Transgenic MMP-2 expression induces latent cardiac mitochondrial dysfunction. Biochem. Biophys. Res. Commun. 358, 189-195. 